https://doi.org/10.15407/ujpe66.5.456

\title{
GENNADII MYKHAILOVYCH ZINOVJEV (to the 80-th anniversary of his birth)
}

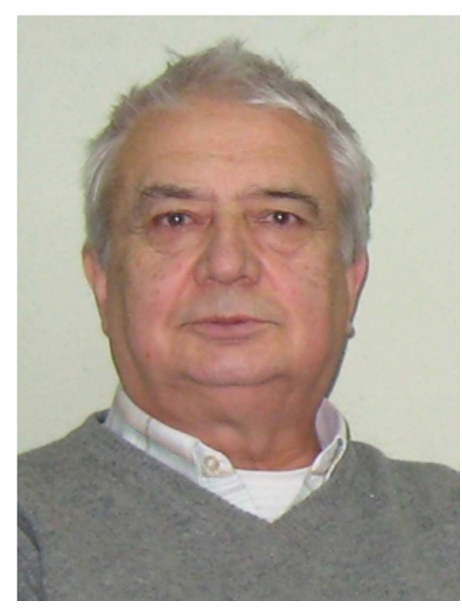

On April 18, 2021, Gennadii Mykhailovych Zinovjev who is the well-known physicist-theorist, a Corresponding Member of the NAS of Ukraine, and the Head of the Department of High Energy Densities at the M.M. Bogolyubov Institute for Theoretical Physics of the NASU has celebrated the 80-th anniversary of his birth.

Gennadii Mykhailovych got the higher education at the Faculty of Physics of the Dnipropetrovsk State University and a Branch of the M.V. Lomonosov Moscow State University at the Joint Institute of Nuclear Research (JINR) in a city of Dubna. After the post-graduate course terminated in 1967 and the defense of the $\mathrm{PhD}$ thesis at the Laboratory of Theoretical Physics of JINR, G.M. Zinovjev began to work at the Institute for Theoretical Physics of the NASU. He started the scientific work at the Department of Elementary Particles headed by Vitalii Petrovych Shelest.

The first scientific studies executed by G.M. Zinovjev that became the basis of its $\mathrm{PhD}$ thesis dealt with the multiple production of hadrons and were related to the development of statistical methods for the description of multiparticle systems. In Kyiv, he advanced some new ideas concerning the statistical properties of the strongly interacting matter in the framework of the so-called dual resonance model. These studies established that the mass spectrum within this model increases exponentially with the mass of hadrons. The same behavior of fireballs in the model of statistical bootstrap was independently proposed by R. Hagedorn in 1965. These two models became the object of studies in the group headed by G.M. Zinovjev. The results obtained in that time were of significant importance for the interpretation of experimental data in the high-energy physics and for the formation of new fundamental theoretical hypotheses. It is sufficient to remember the theory of strings which appeared as a mathematical development of the operator formalism of the dual resonance model. The scientific results obtained by G.M. Zinovjev with co-authors for 1970-1974 were published in the two-volume book by V.P. Shelest, G.M. Zinovjev, and V.A. Miransky "Models of Strongly Interacting Elementary Particles" [in Russian], Atomizdat, Moscow, Vol. 1 (1975), Vol. 2 (1976) and formed the basis of the Doctoral dissertation defended by G.M. Zinovjev in 1975.

In 1977, G.M. Zinovjev and M.I. Gorenstein made a new step in the study of properties of the strongly interacting matter. Their article in the journal "Physics Letters B" proposed a fireball model of the cumulative effect in the processes of collisions of relativistic protons with atomic nuclei. This model opened new possibilities of fundamental importance for the investigation of properties of the hadron states with great masses. It should be emphasized that this article is one of the first theoretical works in the high-energy physics, which indicated that the atomic

ISSN 2071-0194. Ukr. J. Phys. 2021. Vol. 66, No. 5 
nucleus is a significant participant in the process of generation of hadrons. In several years, the processes of relativistic nucleus-nucleus collisions became the object of many experimental programs aimed at the search for signals of the formation of a new form of matter, namely, the quark-gluon plasma (QGP). Such studies are carried on at the Brookhaven National Laboratory in the USA and on the Super Proton Synchrotron (SPS) in CERN.

At the end of the 1970s - the beginning of the 1980s, members of the group headed by G.M. Zinovjev obtained the significant physical results on the statistical mechanics of the hadron matter that received a wide international recognition. One of the principal achievements is the construction of an exactly solvable model of the deconfinement phase transition. In the work by M.I. Gorenstein, G.M. Zinovjev, and V.K. Petrov published in the journal "Physics Letters B" in 1981, the authors considered the quarkgluon bags as universal objects of the hadron matter. Those objects became a base for the description of the phase of hadrons, their resonance states at low energy densities, and the phase of deconfined quarks and gluons (QGP), when the energy density exceeds some critical value. In the works by G.M. Zinovjev and K.A. Bugaev, the statistical model of the quarkgluon bags was further developed with regard for such physical effects as the surface tension and the boundedness of the lifetime of massive bags, which enabled them to expand the field of applications of the model. The works of Zinovjev's group turned out at the center of a new scientific trend and became a push for the study of the strongly interacting matter under extreme conditions with the purpose to find the evidence of the formation of QGP.

In 1985, the Department of High Energy Densities at the M.M. Bogolyubov Institute for Theoretical Physicd of the NASU was organized, and Gennadii Mykhailovych Zinovjev was elected its head. This event became the start of the development of a new direction of physics in Ukraine. Almost all scientists of the new department were disciples and adherents of Professor G.M. Zinovjev. Since that time, many significant scientific results were obtained under his guidance, and the achievements of the department in some trends of the theoretical physics had the priority significance and were highly appreciated by the international scientific community. Without any overstatement, we can say that the citation of the works by G.M. Zinovjev and his colleagues is sufficiently high among the scientific community. Since the very start of their activity, the scientists of the department reveal the intense cooperation with many international centers and experimental collaborations that investigate the collisions at relativistic energies.

Since the middle of the 1980s, the theory of quantum chromodynamics (QCD) on a lattice became one of the significant directions developed in the department. G.M. Zinovjev and his disciple O.A. Borisenko proposed new order parameters, advanced the hypothesis about the role of a triplicity in the confinement mechanics, and formulated the assumption of the interrelation of the deconfinement phase transition and a change in the mechanism of shielding of the triplicity of quark fields.

The formalism of stochastic averaging over strong gluon fields that was developed by G.M. Zinovjev allowed him to make the detailed analysis of Hamilton models and to study the transition with a recovery of the chiral symmetry. It is worth to mention the new results obtained in the department that concern the hydrodynamic model of nucleus-nucleus collisions. For the first time, this model was applied to the analysis of the production of hadrons with high transverse momenta at the stage of hydrodynamic expansion of matter at high temperatures. A number of Zinovjev's recent works are devoted to the development of the model of synchrotron radiation arising due to the interaction of quarks with the collective confinement field in the collision of relativistic heavy ions. In these works, the spectrum of photons with high transverse momenta was calculated and compared with experimental data. The conclusion about a possibility to register such radiation at the production of lepton pairs in corresponding experiments was made. In the recent works by G.M. Zinovjev and Yu.M. Sinyukov, the analysis of the production of photons on various stages of relativistic nucleus-nucleus collisions is performed. It is shown that the experimental data obtained at the Large Hadron Collider (LHC) indicate the necessity to consider the additional mechanism of photonic radiation in the process of hadronization of QGP.

Observing the theoretical results obtained by G.M. Zinovjev with co-authors, it is of interest to remember the fate of a long-standing work on a generalization of parton distributions to the multiparticle case. It was published in "Physics Letters B" in 
1984 and was not noticed for a rather long time. But at the beginning of the $2000 \mathrm{~s}$, it became obvious that this work created a new powerful trend in the physics of many-parton collisions that is intensively developed now in experiments and theoretical studies. This work was cited in a lot of publications, and the proposed trend is the theme of regular international conferences.

G.M. Zinovjev is not only a famous Ukrainian physicist-theorist recognized at the international level due to his pioneer studies in the hadron matter physics and the relativistic nuclear physics. He is one of the founders of the international collaboration ALICE at the LHC in CERN, one of the authors of the project of collider NICA whose construction will be finished soon at JINR (Dubna), and one of the initiators of the creation of the Ukrainian national grid cluster. Due to G.M. Zinovjev, many Ukrainian scientists have got a possibility to become members of collaboration ALICE and to take participation in the experiments that are, possibly, the most complicated for the whole history of the development of science. It should be noted that the introduction of the grid-technology in Ukraine and the first steps of its development are a result of the close collaboration with CERN. Now, the area of applications of the gridtechnology is intensively expanded and includes new scientific trends, applied tasks, and whole industrial branches.

Our sincere wishes of the long life, great happiness, strong health, new achievements, and successes in all affairs to Gennadii Mykhailovych!

A.G. ZAGORODNY, YU.I. IZOTOV, V.M. LOKTEV, V.YU. STORIZHKO, M.F. SHUL'GA, V.P. GUSYNIN, B.I. LEV, E.A. PASHITSKY, E.G. PETROV, YU.A. SITENKO, M.I. GORENSTEIN, E.S. MARTYNOV, YU.M. SINYUKOV, V.V. KUKHTIN 